\title{
As redes sociais na perspectiva de pessoas com o diagnóstico de esquizofrenia em tratamento em um CAPS de Campinas e as implicações
}

no recovery

\section{Social networks by the perspective of people with the diagnosis of schizophrenia in treatment in a "CAPS" from Campinas and the implications in recovery}

\author{
Monica Krieck Montanher ${ }^{1}$, Erotildes Maria Leal ${ }^{2}$ \\ http://dx.doi.org/10.11606/issn.2238-6149.v24i3p183-90
}

Montanher MK, Leal EM. As redes sociais na perspectiva de pessoas com o diagnóstico de esquizofrenia em tratamento em um CAPS de Campinas e as implicações no recovery. Rev Ter Ocup Univ São Paulo. 2013 set.-dez.;24(3):183-90.

RESUMO: O modelo de assistência preconizado pela Reforma Psiquiátrica introduz um cuidado em saúde mental baseado em estratégias de reabilitação psicossocial das pessoas com transtornos mentais. Nesse sentido, as redes sociais podem ajudar os sujeitos a lidarem com a doença. Assim, a análise das redes sociais, a partir da experiência dos sujeitos, pode indicar de que forma essas redes contribuem (ou não) para o processo de recovery. Este artigo apresenta os resultados de uma pesquisa qualitativa informada pelo referencial da Análise Fenomenológica Interpretativa. Teve como objetivo conhecer como as pessoas com diagnóstico de esquizofrenia em tratamento em um CAPS da cidade de Campinas compõem e experimentam as suas redes sociais e que implicações as redes sociais tem no seu processo de recovery. Resultou-se da análise a identificação de categorias temáticas referentes aos vínculos e tipos de interação estabelecidos pelos participantes. Concluiu-se a partir das narrativas que as redes são positivas para a experiência de recovery, se vivenciadas com alguma capacidade de proporcionar esperança, incentivo e suporte. Uma relação será experiência da positivamente se for capaz de minimizar ou ressignificar uma relação ruim ou a própria relação garantindolhe plasticidade.

DESCRITORES: Esquizofrenia; Experiência do adoecimento; Redes sociais; Estado de retorno; Saúde mental; Centros de reabilitação.
Montanher MK, Leal EM. Social networks by the perspective of people with the diagnosis of schizophrenia in treatment in a "CAPS" from Campinas and the implications in recovery. Rev Ter Ocup Univ São Paulo. 2013 set.-dez.;24(3):183-90.

\begin{abstract}
The preconized assistance model by the Psychiatric Reform introduces a mental health care strategies based on psychosocial rehabilitation of people with mental disorders. According to this, social networks can help individuals to handle the disease. Thus, the social network analysis, from the individuals experience, may indicate how these networks contribute (or not) to the process of recovery. This article presents the results from a qualitative research informed by the Interpretative phenomenological analysis. Aimed to know how people with schizophrenia under treatment in a CAPS of Campinas compose and experience their social networks and which impact the social network has in their recovery process. It was resulted from the analysis of thematic category identification related to the bonds and types of interaction established by the participants. It was concluded, based on the narratives, that networks are positive to the recovery experience, if the experience is able to provide hope, encouragement and support. A relationship or bond will be positively experienced if it is able to minimize or reframe a bad relationship or the relationship itself assuring the plasticity.
\end{abstract}

KEYWORDS: Schizophrenia; Ilness experience; Social networking; Recovery; Mental health; Returning state; Rehabilitation centers.

Este trabalho é parte integrante da dissertação de Mestrado intitulada As redes sociais na perspectiva de pessoas com o diagnóstico de esquizofrenia em tratamento nos centros de atenção psicossocial: implicações para o recovery, que foi apresentada ao Programa de PósGraduação em Saúde Coletiva da Faculdade de Ciências Médicas da Universidade Estadual de Campinas, para obtenção do título de mestra em Saúde Coletiva.

1. Mestre em Saúde Coletiva pela Universidade Estadual de Campinas.

2. Doutora em Psiquiatria e Saúde Mental. Professora adjunta do Curso de Medicina da Universidade Federal do Rio de Janeiro - Campus Macaé e Professora Associada da pós-graduação em Saúde Coletiva da Faculdade de Ciências Médicas da Universidade Estadual de Campinas.

Endereço para correspondência: Depto. de Saúde Coletiva, Faculdade de Ciências Médicas, Universidade Estadual de Campinas, CEP: 13083-970,Campinas, SP, Brasil.E-mail: Monica_krieck@hotmail.com; eroleal@gmail.com 


\section{INTRODUÇÃO}

assistência psiquiátrica brasileira sofreu
significativas mudanças no que se
refere à natureza do cuidado prestado aos sujeitos com transtornos mentais nos últimos anos. $\mathrm{O}$ redirecionamento do modelo assistencial em saúde mental vem no sentido de construir uma rede de cuidado que inclua a experiência da loucura e que garanta a efetiva inclusão social das pessoas com transtorno mental, já que as práticas asilares causavam rupturas sociais na vida desses sujeitos.

Para Fontes ${ }^{11}$ a possibilidade de reinserção social da pessoa com transtorno mental, proporcionada pela reforma nos sistemas de atenção, integra toda sua rede social no seu processo de tratamento, possibilitando a reconstrução de um cotidiano que foi perdido pelo sofrimento psíquico. Assim, torna-se fundamental considerar a vida dos sujeitos, tanto quanto a sua doença entendida em termos sinais e sintomas clínicos: "o cuidado em saúde mental amplia-se no sentido de ser também uma sustentação cotidiana da lida diária do paciente, inclusive nas suas relações sociais"24 (p.40).

Nos diversos campos da ciência e, sobretudo na saúde tem havido uma ampliação e crescimento no debate sobre a temática das redes sociais a partir de diferentes perspectivas, interesses e aplicações ${ }^{3,15,16}$.

$\mathrm{Na}$ saúde, o conhecimento das redes sociais das pessoas com transtorno mental pode contribuir para viabilizar novas possibilidades de sociabilidade aos sujeitos. Além disso, é importante conhecer as redes sociais dos sujeitos em sofrimento psíquico, na medida em que nelas as intervenções terapêuticas ocorrem ${ }^{17}$. Somase a isso a contribuição das redes sociais dos sujeitos no processo de recovery dessas pessoas, já que este é um processo dinâmico que sofre influência dos contextos e das relações e interações entre os indivíduos 9 .

As redes sociais têm sido estudadas principalmente a partir de pesquisas qualitativas interessadas em analisar a estrutura e a função dessas redes. No presente estudo, interessa-nos conhecer como essas pessoas compõem e experimentam as suas redes sociais e que implicações as redes sociais tem no seu processo de recovery, ou seja, em que medida as relações experimentadas por eles, os ajuda ou não a manejar e superar o adoecimento mental. Para isso, as redes sociais serão aqui estudadas a partir das experiências vividas e compartilhadas pelas pessoas com transtornos mentais nas suas redes, buscando o que é vivenciado nessa experiência.

O objetivo desse artigo é analisar as redes sociais de pessoas com diagnóstico de esquizofrenia em tratamento em um CAPS da cidade de Campinas. Para análise dos dados, utilizaremos o método fenomenológico interpretativo.

A seguir apresentaremos brevemente o conceito de experiência do adoecimento e a noção de esquizofrenia a partir da perspectiva da psicopatologia fenomenológica, conceitos que direcionaram nosso olhar para estudar as redes sociais na perspectiva dos participantes da pesquisa. Revisitamos também os conceitos de rede social e recovery, partindo do pressuposto de que existe uma relação entre ambas que vale ser analisada.

\section{Experiência do adoecimento}

Estudos sobre experiência do adoecimento ganham destaque na antropologia médica a partir dos anos 1970, devido às mudanças no discurso antropológico sobre as doenças e a insatisfação com o reducionismo biológico.

A experiência do adoecimento (Illness) referese ao modo como os sujeitos, e todos os membros da sua rede social (família, amigos, entre outros) percebem, interpretam e vivenciam o processo de adoecimento. Nesse sentido, a categoria experiência do adoecimento tem um caráter intersubjetivo e corporeificado ${ }^{1}$, interessando-se não apenas pelo modo como os sujeitos representam o seu adoecimento Os aspectos sociais e simbólicos das doenças são importantes, e "a experiência da doença é uma parte íntima dos sistemas sociais de significado e regras de comportamento sendo fortemente influenciada pela cultura" 14 (p.252). No entanto, a análise dos processos saúde-doença vivenciados socialmente, precisam estar articulados com a experiência daquele que a experiência ${ }^{25}$. A experiência do adoecimento, em sua abordagem fenomenológica, supõe uma permanente conexão entre os aspectos biológicos, culturais e sociais, já que o próprio viver é um processo em constante transformação que perpassa todas estas dimensões ${ }^{1}$.

\section{A esquizofrenia a partir da perspectiva da psicopatologia fenomenológica}

Partimos aqui do entendimento que pessoas com esquizofrenia vivenciam algumas experiências relacionadas ao seu modo de ser no mundo que não são encontrados nos manuais diagnósticos atuais, preocupados exclusivamente com a dimensão descritiva dos sintomas. Esta forma não hegemônica de caracterizar a esquizofrenia não toma apenas a dimensão descritiva da sintomatologia e do curso da doença para defini-la, mas leva em conta certas experiências 
particulares de vida e do adoecer que pessoas com este diagnóstico experimentam.

$\mathrm{Na}$ compreensão da esquizofrenia a partir da perspectiva da psicopatologia fenomenológica os sintomas são compreendidos enquanto fenômenos que expressam esta outra forma de relação com o mundo. Tatossian ${ }^{23}$ ressalta que as especificidades psicopatológicas advêm das manifestações do vivido e não se referem às modificações do comportamento. Autores desta tradição fenomenológica Minkowski, Blankenburg, Parnas, dentre outros ${ }^{18,21}$ - indicam que este modo de relacionamento com o mundo não pode ser observado simplesmente por meio dos sinais e sintomas que usualmente caracterizam a esquizofrenia. Nesta perspectiva haveria uma alteração básica que é anterior e decorre de desordem que envolve aspectos relacionados ao que Bleuler denominou de "autismo Minkoswki e Blankenburg definem o fenômeno nomeado por Bleuler de autismo como um modo particular de experimentar o mundo que decorre de perturbações da ipseidade ou do self $^{18}$. Estas perturbações se dariam em campos: no âmbito da consciência de si préreflexiva, tácita, cursando com à diminuição da autoafecção (self-affection) ou sensação de vitalidade; e no âmbito da consciência reflexiva, onde a alteração se manifestaria através da hiperreflexividade, compreendida como uma forma exagerada de autoconsciência ${ }^{21}$.

\section{As redes sociais: uma contextualização}

Pesquisas sobre redes sociais vêm sendo desenvolvidas na sociologia, na antropologia e até mesmo na saúde ${ }^{3}$. As redes sociais podem ser definidas, a partir destes estudos, como a somatória das relações estabelecidas por um indivíduo que são percebidas por ele como significativas diferenciando-se assim do restante da sociedade $^{22}$. Os sujeitos sentem-se, em alguma medida, pertencentes e reconhecidos em suas redes sociais, que contém, sustentam e são geradas por histórias que constituem a identidade de seus membros. Portanto, estar na vida com outras pessoas em suas redes é um processo sem fim de construção do self e dos outros ${ }^{22}$.

A estrutura de uma rede social tem duas características principais: o tamanho e o padrão de ligação. $\mathrm{O}$ tamanho da rede representa o número de pessoas que compõem a rede de um indivíduo. O padrão de ligação refere-se à forma de conexão estabelecida entre os membros de uma rede, isto é, podem ser tanto ligações diretas, que ligam um dado indivíduo diretamente a outro, quanto conexões indiretas, que ligam o indivíduo a pessoas que não estão diretamente conectadas a ele, como amigos dos amigos que ampliam as relações estabelecidas pelo indivíduo ${ }^{12}$.
Assim, as redes sociais incluem todo conjunto de vínculos interpessoais do indivíduo ${ }^{22}$.

Pertencer a uma rede requer que seus membros participem ativamente dos processos que nela ocorrem. Entretanto, de acordo com Muramoto e Mângia ${ }^{16}$, um sujeito adoecido muitas vezes não dá conta dessa participação o que enfraquece sua interação, acarretando em um empobrecimento em seus laços sociais.

Alguns estudos que falam sobre rede social e saúde compreendem que o cuidado baseia-se na premissa de que saúde é um "fenômeno social e produto das interações humanas" $" 15$ (p.27). Dois tipos de evidências tem destaque nos estudos que discutem a relação saúde/ rede social. Uma sugere que a boa qualidade de ligações no interior das redes traz uma perspectiva de melhorias nas condições de saúde ao promover apoio social, troca de experiências, agilidade na utilização dos serviços de saúde, entre outros. A outra indica que redes sociais empobrecidas de relações ou que não estabelecem influências positivas entre seus membros constituem um risco à saúde s5,16,20,22 $^{2}$

\section{Algumas considerações sobre redes sociais e transtorno mental}

O termo rede social aparece pela primeira vez na literatura clínica da esquizofrenia, em um artigo sobre "terapia de rede" de Speck e Rueveni. Na realidade, o estudo das redes sociais na esquizofrenia responde ao crescente interesse de caracterização do mundo social desses sujeitos ${ }^{3}$.

Compreende-se, então, que o fortalecimento das redes sociais das pessoas com transtorno mental é necessário, pois o cuidado em saúde mental pressupõe que as experiências de vida dos sujeitos e não exclusivamente a sua doença, devem ser considerados. Além disso, de acordo com estudos realizados por Pattinson e Pattison ${ }^{19}$, na esquizofrenia as experiências de constituição de rede são diferentes, pois além dos esquizofrênicos possuírem uma quantidade limitada de pessoas em sua rede, não incluindo relações de longo prazo, estes exibem um sistema fechado de relações.

Diversos estudos mostram também a correlação existente entre o empobrecimento e fragilidade das redes sociais e a questão da internação $0^{13,16,20}$. Pesquisas demonstram que egressos de hospitais psiquiátricos relatam dificuldade de interação com as pessoas a sua volta $^{20}$. Além disso, usuários que tiveram sua primeira internação conseguiram formar redes mais sólidas e amplas do que aqueles usuários com longos períodos de institucionalização ${ }^{16}$. Há também um aumento do número 
de profissionais de saúde e colegas de serviços de saúde mental nas redes de usuários institucionalizados ${ }^{13}$. Na realidade, as pessoas com doenças mentais graves de um modo geral estão mais propensas a terem profissionais de saúde na composição de suas redes. O fato é que esses profissionais ou serviços exercem a função de apoio instrumental e emocional.

A concepção de rede social apresentada até aqui defende que uma rede social empobrecida de relações sociais é algo negativo, pode acarretar danos à saúde, indica maior fragilidade relacional e possível falta de apoio social, o que uma rede consolidada seria capaz de proporcionar. No entanto, Corin e Guilles ${ }^{4}$, a partir do desenvolvimento de metodologias que exploram as experiências subjetivas dos participantes de suas pesquisas, traz o conceito de "retirada positiva", que imprime outro significado a rede social. Estudando narrativas de pessoas com esquizofrenia, a autora percebeu que algumas destas pessoas experimentavam negativamente a rede social ampliada. Certo retraimento da rede social, portanto, podia ser vivenciado como um fator protetivo. Ela também observou que algumas das pessoas que atribuíam a sua rede um valor positivo, constituíam uma ligação com seus membros que para um observador externo pareceria uma ligação frouxa e pouco intensa.

DeVries e Delespaul ${ }^{8}$, também fizeram referência a este outro modo de vivenciar as redes. Estes autores perceberam, a partir das experiências dos sujeitos adoecidos, que estar ou não sozinho pode ser uma escolha ativa, que ocorre como uma estratégia de enfrentamento ao cotidiano.

Enfim, o fortalecimento das redes sociais das pessoas com transtorno mental precisa considerar a experiência dos sujeitos. As estratégias para se trabalhar com as redes não devem ser prescritivas, já que não existe um único padrão de constituição de laços sociais que seja adequado à melhoria das condições de saúde.

\section{Recovery na saúde mental}

A definição para o recovery não é única, pois é influenciada por fatores multidimensionais que perpassam a vida do sujeito e pelo contexto no qual esse sujeito se insere ${ }^{2,9}$. Na literatura encontramos duas dimensões diferentes para conceituar o recovery. Uma delas está mais vinculada ao modelo biomédico, pois se refere à diminuição dos sintomas e restauração das condições cognitiva, social e funcional. A outra está mais ligada ao modelo de atenção psicossocial que se baseia no indivíduo e na perspectiva de que é possível ter uma vida significativa mesmo diante de uma doença severa e persistente 6 .

Ambas as concepções de recovery se opõem a uma forma bastante pessimista de conceber a esquizofrenia, pois não pressupõem a remissão total dos sintomas e nem a cura, e reconhece que pessoas em intenso sofrimento psíquico podem ter uma vida para além da doença ${ }^{2,9}$.

Estamos aqui interessados na concepção de recovery ligada ao modelo de atenção psicossocial, que parte da experiência de quem vive o adoecimento. Nesta perspectiva o recovery é um processo dinâmico e complexo que reforça a capacidade do sujeito de viver uma plena e produtiva, apesar de sua doença. Significa uma mudança de atitude pessoal diante do adoecimento ${ }^{2,7}$.

$\mathrm{O}$ recovery pode ocorrer mesmo sem intervenção profissional, ainda que seja papel dos profissionais e dos serviços dar apoio aos usuários e assim, contribuir (ou não) para o processo do recovery ${ }^{2}$. Ocorre que muitas vezes os profissionais lidam com a experiência do adoecimento de uma forma reducionista e tutelada, o que reforça a necessidade de reformulação do papel e das práticas dos profissionais e dos serviços?.

É preciso valorizar também as experiências e interesses dos usuários na integração com sua comunidade e com sua rede social, afinal, o recovery envolve uma dimensão social, pois ocorre na relação com os outros, por meio da participação social.

\section{METODOLOGIA}

Este artigo é um recorte da dissertação de mestrado intitulada "As redes sociais na perspectiva de pessoas com o diagnóstico de esquizofrenia em tratamento nos centros de atenção psicossocial: implicações para o recovery", apresentada ao Programa de Pós-Graduação em Saúde Coletiva do Departamento de Saúde Coletiva da Faculdade de Ciências Médicas da Universidade Estadual de Campinas (UNICAMP). É um estudo informado pelo quadro de referência da Análise Fenomenológica interpretativa, e parte de uma pesquisa mais ampla intitulada "Experiência, narrativa e conhecimento: a perspectiva do psiquiatra e a do usuário". A pesquisa maior, do qual esta dissertação é parte, foi realizada de 2009 a 2011, desenvolvida nas cidades do Rio de Janeiro, Salvador e Campinas em parceria entre a UNICAMP, a Universidade Federal do Rio de Janeiro (UFRJ) e a Universidade Federal da Bahia (UFBA). Foi aprovada pelo CEP do Departamento de Medicina Preventiva e Social da UNICAMP - parecer $n^{\circ}$ 870/2009 e pelo CEP da Secretaria Municipal de Saúde do Rio de Janeiro - parecer $\mathrm{n}^{\mathrm{o}} 210^{\mathrm{a}} / 2009$ e teve financiamento do CNPQ. Teve como objetivo investigar se e como o 
conhecimento técnico científico dos psiquiatras, presente na construção do diagnóstico e do projeto terapêutico, se refletia na experiência vivida dos usuários acerca do processo saúde-doença-cuidado e, também examinar se a experiência de adoecimento dos usuários era capaz de iluminar o conhecimento técnico do psiquiatra e como isso se dava.

Para coleta dos dados foram realizados grupos focais áudio gravados que ocorreram em três tempos, para cada um dos grupos de participantes (psiquiatras e usuários), organizados da seguinte forma: discussão do processo de formulação do diagnóstico e projeto terapêutico com os psiquiatras e das experiências do processo de adoecimento - abordadas a partir dos antecedentes da primeira crise, do momento da crise, do processo de tratamento e do entendimento de como o próprio sujeito, seus familiares, amigos e sociedade lidam com o problema - com os usuários, no tempo 1; apresentação das narrativas dos usuários aos psiquiatras e vice-versa, no tempo 2; discussão das narrativas produzidas nos dois primeiros tempos em grupos mistos de psiquiatras e usuários, no tempo 3 .

$\mathrm{O}$ tema das redes sociais, não foi foco da pesquisa maior, mas foi tema emergente da fala dos usuários sobre o processo de adoecimento, demonstrando a relevância desta temática para experiência vivida dos usuários. Portanto, nesta pesquisa foram utilizadas as narrativas dos usuários, produzidas no primeiro tempo.

\section{Critérios para seleção dos usuários}

O recrutamento dos usuários participantes da pesquisa matriz, autores das narrativas, objeto deste estudo, se deu a partir de quatro etapas: 1) auto-atribuição de experiências relacionadas aos transtornos do espectro esquizofrênico, mediante apresentação de um vídeo com auto-relatos de pessoas com esquizofrenia dramatizado por atores; 2) atribuição do diagnóstico para transtorno do espectro esquizofrênico pela equipe dos CAPS e pelo instrumento diagnóstico MINIPLUS (Mini Internacional Neuropsychiatric Interview); 3) estar em tratamento no CAPS; 4) aceitar participar da pesquisa.

Após a inclusão do usuário no estudo, ele foi devidamente informado sobre os objetivos e procedimentos do estudo, através da leitura do Termo de Consentimento Livre e Esclarecido (TCLE).

Este estudo se debruçou sobre as narrativas produzidas no grupo focal com usuários de Campinas, do qual participaram 4 pessoas. No quadro 1 (em anexo) apresentamos o perfil sócio-demográfico de cada participante.

Quadro 1 - Perfil sócio-demográfico dos participantes da pesquisa

\begin{tabular}{|l|c|l|l|l|l|}
\hline & Idade & Raça & Estado Civil & Grau de instrução & Atividades de trabalho \\
\hline Cícero & 33 & Branco & Solteiro & Ensino médio incompleto & Aposentado pelo INSS \\
\hline César & 35 & Branco & Solteiro & Ensino médio incompleto & $\begin{array}{l}\text { Exerce trabalho informal e recebe o } \\
\text { Benefício da Prestação Continuada } \\
\text { (BPC) }\end{array}$ \\
\hline Caio & 41 & Branco & Solteiro & Ensino médio incompleto & Aposentado pelo INSS \\
\hline Cleuza & 50 & Branca & Divorciada & Ensino Fundamental Incompleto & Desempregada \\
\hline
\end{tabular}

\section{RESULTADOS}

Baseando-se no método fenomenológico interpretativo utilizado, cinco categorias temáticas foram identificadas a partir dos diferentes tipos de interação e vínculos constituídos pelos participantes. São elas: família; igreja/comunidade religiosa; profissionais/serviços de saúde; relações ocasionais; "retirada positiva".

\section{Família}

Nas narrativas sobre família, evidencia-se uma vivência familiar extremamente variável quando se trata do processo de adoecimento. Relatos demonstram de um lado que a família pode servir de apoio no manejo com a doença, de outro ela pode ser vivenciada como desencadeadora do processo de adoecimento. O desprezo e abandono da família são identificados como consequência do adoecimento como exemplificam os fragmentos abaixo:

[...] quando a família descobriu, nós que temos essa parte de esquizofrenia, que tivemos um problema mental, a familia oficial resolveu nos abandonar. (César)

Porque eu não tinha namorada [...] sempre que eu chego perto de uma moça pra conversar, ela chega e minha mãe fala: "é frouxo" e depois fica brigando e fala: "ele é frouxo" (Cícero) 
No trecho mostrado a seguir, percebe-se que um mesmo familiar exerce papeis diferentes ao longo do tempo. Este familiar dedica atenção e apoio em um momento, mas causa sofrimento em outro.

A crise que eu tinha já aconteceu também [...]meu irmão me levou na PUC lá, e eu fiquei bom, deram uma injeção em mim, e eu fiquei bom. (Caio)

E como é que você ficava pra eles te levarem lá pra internação? O que eles diziam pra você? (moderador)

\section{[...] Porque é muita perturbação com meu irmão, ele reclama de tudo sabe? acordar de manhã cedinho e comprar pãozinho e ele vai atrás, mandar. (Caio)}

\section{Igreja/Comunidade religiosa}

A igreja e a comunidade religiosa, de modo geral, aparecem como elementos importantes da rede social. No entanto, a dinâmica e função que esse componente possui na rede também não é única. A igreja, para alguns, constitui espaço de continência e prevenção de uma nova crise ou proporciona uma melhora na condição de adoecimento.

Eu vou continuar indo, porque lá é mais estudo que a gente tem $[\ldots]$ (César)

Meu irmão me leva na igreja para ler uma bíblia, para evitar o mal [...] (Caio)

As crenças religiosas também podem exercer certa função na rede social. No trecho abaixo, o usuário refere ser "controlado" cotidianamente por entidades religiosas. Um controle paradoxal, todavia, na medida em que está de alguma forma submetido aquilo que ele deseja.

Estou sendo guiado por um monte de macumbeiros, feiticeiros, pelo seguinte motivo, eles tem falado para mim o seguinte "que horas você quer levantar?" [...] se eu digo que é sete horas, chega sete horas eu não consigo dormir mais, se for oito horas, oito horas eu não consigo dormir mais, mesmo tomando remédio.(César)

\section{Profissionais/Serviços de Saúde}

Outro elemento da rede social referido nas narrativas está relacionado aos serviços de saúde mental. Nos relatos é possível notar que o CAPS e os profissionais de saúde, constituem a rede social desses usuários ao cumprirem a tarefa de auxiliar no manejo e propor estratégias de enfrentamento da doença. Em muitos momentos esses profissionais tornam-se responsáveis por administrarem situações de vida de um modo geral. Nesse sentido, o CAPS parece desempenhar a função de um lugar estratégico para lida de momentos de maior sofrimento.

[...] O CAPS que está tomando conta da minha vida [...] e não tem ninguém para resolver meus problemas a não ser aqui [...] (Cleusa).

Outras narrativas demonstram que outras conexões são estabelecidas no espaço institucional, como relações de amizade ou relações ocasionais, que podem ser constituídas a partir de atividades várias, formais e estruturadas ou não, dentre elas uma refeição feita coletivamente, um grupo, etc.

E vir pro CAPS ajuda mais no que, gente? (Moderador)

$$
\begin{aligned}
& \text { A gente conversa! (Cleusa) } \\
& \text { Eu venho pra comer. (Cícero) }
\end{aligned}
$$

Jogar bola, fazer terapia, ter amizade com um, fazer comunicação com o outro... (Caio)

\section{Relações Impessoais}

Apresentam-se relevantes também nas redes sociais dos usuários aquelas relações que ocorrem casualmente e são impessoais. A constituição deste tipo de laço social parece acontecer pela necessidade de apoio social em situações específicas ou para constituição de outros laços afetivos que podem não ser estabelecidos no contato com sua rede social mais direta. Observou-se que as motivações para o estabelecimento de laços com estas características são diversas: necessidade de apoio social em determinadas situações de vida, desejo de ser suporte social para outras pessoas, ou até mesmo para construção de laços afetivos/amorosos.

[...] ai a minha cabeça já dá um branco, ai já não sei onde eu estou mais. Ai a policia tem que me trazer para minha casa, porque na minha cabeça eu não to conseguindo nada [...] e quando eu vejo pessoas na rua eu tenho medo, que eu to ameaçada. Ontem eu liguei três vezes para a polícia, mas não resolve e eu não sei o que fazer (Cleusa)

Isso é lá no bairro onde você mora? (Moderador)

No bairro... Conversando. (Caio)

A constituição de novas conexões nem sempre, nas narrativas estudadas, foi vivenciada de maneira positiva. No trecho da narrativa de um dos usuários abaixo apresentada, a sociedade, segundo ele, parece não contribuir para possibilidade de constituição de novas redes.

[...] eu fiz isso tudo pra ganhar direito e que direito eu vou ganhar se eu perco? Assim fica dificil estudar, 
trabalhar, fica dificil interagir com a sociedade que só cobra e não atribui. Então eu acho [...] que fica buscando sempre a falha, então vai ser explorada a falha da pessoa ao invés de explorar a qualidade que tem... (César)

\section{"Retirada Positiva"}

Esta última categoria relacionada ao conceito "Retirada Positiva" foi identificada principalmente na narrativa de uma das usuárias. Em vários momentos ela relata uma postura de distanciamento das pessoas, pela preferência de estar sozinha. Esta mesma usuária apresenta-se em diversas ocasiões bastante solícita, no entanto, afirma que ficar sozinha a faz se sentir bem.

Mesmo que esteja sozinha, eu ando em tudo que é lugar. Tem acompanhante, e eu me sinto mal, eu não gosto que ninguém anda comigo. Eu não gosto que ninguém dorme lá no meu quarto também. Porque eu fico nua sentada e fico só meditando nas coisas que estão acontecendo tudo errado [...] (Cleusa)

Quando a gente fica sozinha a gente se sente bem (Cleusa)

\section{DISCUSSÃO}

As categorias identificadas nas narrativas revelaram qual a dinâmica existente nas relações sociais estabelecidas pelos usuários participantes da pesquisa. Com isso, evidenciam-se elementos que contribuem para a compreensão da implicação da rede social no processo de recovery.

A família faz parte da rede social de todos os usuários, porém, o suporte e apoio que pode oferecer dependem da forma como as relações familiares são constituídas. Muitas dessas pessoas experenciam cotidianamente relações familiares intolerantes. As narrativas indicam que um sujeito pode preferir afastar-se da família para se proteger de experiências de mau trato e estigmatização, por exemplo. Neste caso, não ter a família na rede social pode não necessariamente ser a simples expressão de uma deficiência e fraqueza que limita a sua rede, mas uma saída protetiva.

Ainda com relação à família percebeu-se que a doença mental pode provocar uma diversidade de reações nas pessoas, especialmente aquelas do convívio familiar ${ }^{20}$. Como vimos nas narrativas, um mesmo membro da família não estabelece uma relação linear com o usuário. Isso sugere que a rede deve ser sempre considerada em sua dimensão processual e impermanente.

Em relação à igreja as narrativas mostraram que existe um sentimento de que pertencer a um grupo religioso pode ajudar a evitar ou minimizar o sofrimento. Além disso, existe todo um significado simbólico em torno da religiosidade. A estrutura religiosa pode dar às pessoas maneiras mais práticas para estruturar suas experiências ${ }^{4}$.

Vimos também que os serviços de saúde tem uma grande importância na rede social dos usuários, pois diversas relações são ali constituídas. Os usuários muitas vezes não conseguem estabelecer vínculos fora do circuito de saúde. Mas é preciso destacar que alguns tomam o espaço do CAPS como um lugar que lhes possibilita experiências que não necessariamente estão previstas para acontecer ali. A construção de laços de amizade e suporte com outros usuários é um exemplo. No que se refere aos profissionais, observou-se que estes desempenham uma função para além do suporte nos momentos de maior dificuldade. Alguns usuários indicam relação de significativa dependência com profissionais, com baixo grau de autonomia.

As narrativas sobre as relações impessoais vividas pelos usuários revelam que os vínculos com função de suporte não necessariamente são constituídos com pessoas que conhecemos por nome, ou são mais próximas. Nem tampouco nesse tipo de relação os laços estão fortalecidos, mas ainda assim, o apoio existe.

Uma das hipóteses para que este tipo de relação ocorra é porque essas pessoas demandam e cobram menos, já que a cobrança parece ser algo que limita a rede 4 .

Por fim, a "retirada positiva", como proposta por Corin e Lauzon ${ }^{5}$, foi observada nas narrativas estudadas. Segundo Hammer et al. ${ }^{12}$, a retirada pode ser uma reação de auto-proteção contra as tensões de uma vida social intensa e que exige interação entre os indivíduos mesmo frente as dificuldades que as pessoas com transtornos mentais apresentam.

\section{CONCLUSÃO}

A partir da análise do material concluímos que a contribuição que a rede social do sujeito pode dar para o recovery, exige uma análise complexa. Verdades tais como rede empobrecida é necessariamente contrária a recovery ou ausência do núcleo familiar é ruim, ou ainda relações impessoais são sempre pobres, não se sustentam quando confrontado com as narrativas. A potência de uma dada rede para promover recovery exige uma analise quantitativa e qualitativa. Saber, pela perspectiva de quem a vivencia, como os elementos que a compõem se articulam, como são valorados e experienciados e se cumprem em alguma medida a tarefa de proporcionar esperança, incentivo e suporte, mesmo que isso não se dê de forma linear e permanente, é fundamental 9 .

No entanto apenas a simples identificação de vivências positivas ou negativas nas relações estabelecida na rede social não é suficiente para indicar a sua potencia 
para a promoção do recovery. É importante compreender se, quando vivida negativamente em alguma circunstância e se positivamente em outra, esta experiência positiva tem força

\section{Referências}

1. Alves PCB, Rabelo MCM, Souza IMA. Introdução. In: Rabelo MC, Alves PC, Souza IM, editors. Experiência de doença e narrativa. Rio de Janeiro: Editora Fiocruz; 1999. p.11-39.

2. Antonhy WA. Recovery from mental illness: the guiding vision of the mental health service system in the 1990s. Psychosoc Rehabil J. 1993;16(4):11-23.

3. Bells CC. Social network and schizophrenia. Psychiatr Q. 1979;51(3):209-15.

4. Corin E, Guilles L. Réalités et mirage: les espaces psychiques et sociaux de la reinsertion. Rev Santé Mentale Québec. 1988;13(1): 69-86.

5. Corin E, Lauzon G. Positive withdrawal and the quest of meaning: the reconstruction of the experience among schizophrenics. Psychiatry. 1992;55:266-78.

6. Davidson L, Lawless MS, Leary F. Concepts of recovery: competing or complementary? Curr Opin Psychiatry. 2005.

7. Deegan P. Recuperação como uma viagem do coração. Psychiatr Rehabil J. 1995;19(3):1-19.

8. Devries MW, Delespaul AEG. Time, context, and subjective experiences in schizophrenia. Schizophr Bull. 1989;15(2):23344. doi:10.1093/schbul/15.2.233

9. Duarte T. Recovery da doença mental: Uma visão para os sistemas e serviços de saúde mental. Análise Psicol. 2007;25(1):127-33.

10. Estroff SE. Self, identity, and subjective experiences of schizophrenia: in search of the subject. Schizophr Bull. 1989;15(2):189-96. doi:10.1093/schbul/15.2.189

11. Fontes BAM. Redes sociais e saúde: sobre a formação de redes de apoio social no cotidiano de portadores de transtorno mental. Política Trabalho. Revi Ciên Sociais. 2007;26:87-104.

12.Hammer M, Makiesky-Barrow S, Gutwirth LVOL. Social networks and schizophrenia. Schizophr Bull. 1978;4(4):52245. doi:10.1093/schbul/4.4.522

13. Holmes-Eber P, Riger S. Hospitalization and the composition of mental patients' social networks. Schizophr Bull. 1990;16(1):157-64. doi:10.1093/schbul/16.1.157

14. Kleinman A, Eisenberg L, Good B. Culture, illness and care. de minimizar ou ressignificar o que é considerado ruim. Se assim for, estas relações vão contribuir em alguma medida para o processo de recovery.

clinical lessons from anthropologic and cross-cultural research. Ann Intern Med. 1978;88(2):251-58. doi:10.7326/0003-4819$88-2-251$

15. Mângia EF, Muramoto MT. O estudo de redes sociais: apontamentos teóricos e contribuições para o campo da saúde. Rev Ter Ocup Univ São Paulo. 2005;16(1):22-30. doi: http:// dx.doi.org/10.11606/issn.2238-6149.v16i1p31-39

16. Mângia EF, Muramoto MT. A sustentabilidade da vida cotidiana: um estudo das redes sociais de usuários de serviço de saúde mental no município de Santo André (SP, Brasil). Ciên Saúde Coletiva. 2011;16(4):2165-77. http://dx.doi. org/10.1590/S1413-81232011000400016

17. Morin RC, Seldman E. A social network approach and the revolving door patient. Schizophr Bull. 1986;12(2): 262-73. doi:10.1093/schbul/12.2.262

18. Parnas J, Bovet P, Zahavi D. Schizophrenic autism: clinical phenomenology and pathogenetic implications. World Psychiatr. 2002;1(3):131-6.

19. Pattison M, Pattison ML. Analysis of a schizophrenic psychosocial network. Schizophr Bull. 1981;7(1):135-43. doi:10.1093/schbul/7.1.135

20. Salles MM, Barros S. Relações do cotidiano: a pessoa com transtorno mental e sua rede de suporte social. Physis Rev Saúde Coletiva. 2011;21(2):561-79. http://dx.doi.org/10.1590/ S0103-73312011000200012

21. Sass LA, Parnas J. Schizophrenia, consciousness, and the self. Schizophr Bull. 2003;29(3):427-44.

22. Sluzki CE. A rede social na prática sistêmica. 3a ed. São Paulo: Casa do Psicólogo; 2007.

23. Tatossian A. Fenomenologia das psicoses. São Paulo: Escuta; 2006.

24. Tenório F. A reforma psiquiátrica brasileira, da década de 1980 aos dias atuais: história e conceito. História Ciên Saúde. 2002;9(1):25-59. http://dx.doi.org/10.1590/S010459702002000100003

25. Young A. The anthropologies of Illness and Sickness. Annu Rev Anthropol. 1982;11(1):257-85. doi: 10.1146/annurev. an.11.100182.001353 